\title{
Commentary: Compulsive drug use is associated with imbalance of orbitofrontal- and prelimbic-striatal circuits in punishment-resistant individuals
}

\author{
Hang-Bin Zhang, Hui Zheng, Yi Zhang and Di Zhao* \\ Shanghai Key Laboratory of Psychotic Disorders, Shanghai Mental Health Center, Shanghai Jiao Tong University School of \\ Medicine, Shanghai, China
}

Keywords: compulsive behavior, frontostriatal networks, functional connectivity, substances use disorders, self-administration

\section{A Commentary on}

Compulsive drug use is associated with imbalance of orbitofrontal- and prelimbic-striatal circuits in punishment-resistant individuals

by Hu, Y., Salmeron, B. J., Krasnova, I. N., Gu, H., Lu, H., Bonci, A., et al. (2019). Proc. Natl. Acad. Sci. U.S.A. 116, 9066-9071. doi: 10.1073/pnas.1819978116

\section{OPEN ACCESS \\ Edited by: \\ Weili Zhu, \\ Peking University, China \\ Reviewed by: \\ Yan Sun, \\ Peking University, China \\ ${ }^{*}$ Correspondence: \\ Di Zhao}

dizhao_sunset@163.com

Received: 11 June 2020

Accepted: 13 July 2020

Published: 25 August 2020

Citation:

Zhang $H-B$, Zheng $H$, Zhang $Y$ and

Zhao D (2020) Commentary:

Compulsive drug use is associated with imbalance of orbitofrontal- and

prelimbic-striatal circuits in

punishment-resistant individuals.

Front. Neural Circuits 14:49.

doi: 10.3389/fncir.2020.00049
Substance use disorders (SUDs) cause heavy economic burden and seriously challenges public security. Nearly 35 million people are receiving substance abuse treatment, and 585,000 people in the world died from overdose in 2017 (The United Nations Office on Drugs and Crime, 2019). SUDs has been widely associated with disrupted control of urges to use drugs, revealed by compulsive drug-seeking despite harmful consequences (Hyman et al., 2006; Volkow et al., 2016). Over the past two decades, researchers have shifted their focus to investigate chronic substance use induced brain plasticity at neurocircuit level underlying compulsive behaviors in addiction [e.g., cocaine (Hu et al., 2015), nicotine (Bi et al., 2017), alcohol (Grodin et al., 2018; Strosche et al., 2020), heroin (Wang et al., 2013), methamphetamine (Kohno et al., 2014)]. A recent research using resting-state functional connectivity (rsFC) has identified "go"- "stop" circuits imbalance contributing to compulsive drug use symptoms in cocaine dependents. The "go" circuits (the right ventral striatum superior-anterior prefrontal cortex/orbitofrontal cortex) are hypothesized to promote drug seeking, while the "stop" circuits (the right ventral striatum inferior-dorsal anterior cingulate cortex) are hypothesized to restrain such behaviors (Hu et al., 2015). However, a full understanding of "go"- "stop" circuits in three-stage (binge/intoxication, withdrawal/negative affect, and preoccupation/anticipation) addiction cycle is still poorly understood. Furthermore, neurocircuit mechanisms that account for the heterogeneity in drug response in the preclinical study still remain unknown.

These understandings were addressed in a recent study published in the journal of Proceedings of the National Academy of Sciences of the United States of America by Hu et al. (2019). The research employed a longitudinal functional magnetic resonance imaging (fMRI) design to dissect neurocircuit mechanisms underlying addiction in the self-administration (SA) rat model. The animal experimental procedure paralleled the stages of compulsive drug taking cycle in methamphetamine dependents, including SA phase as binge/intoxication stage and foot-shock punishment as negative affect/withdrawal stage. All rats were scanned by fMRI at baseline and after these key time points above. Seed based rsFC analyses were applied later. In consideration of the 
individual difference on compulsive behavior, rats were differentiated to shock-resistant (SR) and shock-sensitivity (SS) subgroup rats by "compulsivity index" (CI, a ratio of meth infusion on last foot shock day versus last SA day). In SA behavior, no difference was found between two subgroup rats before and after stable SA. During punishment phase, the shockresistant (SR) subgroup rats showed continuous lever pressing with higher CI, while the shock-sensitivity (SS) subgroup rats controlled drug use with lower CI. In neurocircuitry level, the rsFC of orbitofrontal-medial striatum (OFC-MS) "go" circuit increased and prelimbic-ventral striatum (PrL-VS) "stop" circuit decreased until the end of SA phase in both two subgroups. While in foot-shock punishment stage, only the rats of SR group showed the rsFC of go circuit decreased and stop circuit increased, which consisted with their SA behavior. Briefly, the balance between go and stop circuit (indicated by go-stop) differed in two subgroups, and significantly associated with compulsive level. These results showed that when facing adverse consequences (e.g., punishment), addictive individuals diversified the compulsive behavior and go-stop circuit.

\section{LIMITATIONS AND FUTURE DIRECTIONS}

Drug addiction is characterized by disruption in finely balanced brain networks. Over the two decades, the animal models (Shippenberg and Koob, 2002) and human neuroimaging studies ( $\mathrm{Hu}$ et al., 2015) has facilitated the understanding of neurobiology of addiction. In a recent review, Koob and Volkow have proposed that three domains (motivational "wanting"incentive salience, emotional "liking"-negative emotional state, and executive control) mediated by three neurocircuits (basal ganglia, extended amygdala, and prefrontal cortex) (Koob and Volkow, 2016). A long stand of human studies had indicated the orbitofrontal cortex (the hub of go circuit) was essential for regulating incentive salience when a drug cue was presented to the drug dependents (Volkow and Fowler, 2000; Clarke et al., 2008; Hart et al., 2014). And evidence from rodent research has demonstrated that drug-related reinstatement involved by stop circuit that links PrL to VS, which correspond with anterior cingulate cortex (ACC) in human (McFarland and Kalivas, 2001). $\mathrm{Hu}$ and colleagues have identified the balance of "go-stop" circuit to produce compulsive-like habits by combining the merits of animal model and brain imaging in individuals. However, in the study, animal model could not fully emulate the addiction cycle (e.g., the preoccupation/anticipation stage, which is a pivotal stage of relapse in humans) and complex emotional dysregulation correlated with withdrawal and protracted abstinence (de Wit,

\section{REFERENCES}

Bi, Y., Yuan, K., Guan, Y., Cheng, J., Zhang, Y., Li, Y., et al. (2017). Altered resting state functional connectivity of anterior insula in young smokers. Brain Imaging Behav. 11, 155-165. doi: 10.1007/s11682-0169511-z

Clarke, H. F., Robbins, T. W., and Roberts, A. C. (2008). Lesions of the medial striatum in monkeys produce perseverative impairments during reversal
2009; McEwen and Morrison, 2013; Maier et al., 2015) in human. There is also evidence indicating withdrawal from drug can cause fatigue, irritability, insomnia, and psychological symptoms (e.g., depression and anxiety; Koob and Le Moal, 1997). Future study to ascertain the shared and distinct neurocircuit mechanisms between depression/anxiety and addiction would be of interest.

In conclusion, this research has suggested that the imbalance of brain circuits plays an important role in maladaptive behavior in drug dependents (Everitt and Robbins, 2016). These findings have underscored that the balance of restingstate functional connectivity would be a potential neuroimaging biomarker to forecast future addiction relapse, and provide neurocircuit candidate for exploring the genetic basis of endophenotypes related to addiction. Moreover, these results extended previous understanding of fundamental brain circuits involved in addiction cycle and opened for a principled way to develop individualized treatment in SUDs. Prior works from our lab have focused on stimulating the dorsolateral prefrontal cortex (DLPFC), a hub of frontal-striatal circuitry that is dysfunctional in SUD individuals (Liu et al., 2017, 2019; Shen et al., 2017; Zhao et al., 2020). Our results have manifested through either activation of left DLPFC or inhibition of the right DLPFC may reduce craving and alleviate symptoms of depression in methamphetamine dependents (Zhao et al., 2020). Another strand studies from Hanlon lab have suggested that stimulating left frontal areas can induce selective changes in ventromedial prefrontal cortex (VMPFC), the striatum, and ACC thus change drug cue reactivity in cocaine dependentand alcohol dependent patients (Hanlon et al., 2015, 2016, 2017, 2018). These empirical results showed that it would be possible to attenuate stop circuit through targeting frontal areas or ACC, which may lay a foundation for future clinical trials for treating SUDs.

\section{AUTHOR CONTRIBUTIONS}

$\mathrm{H}-\mathrm{BZ}, \mathrm{HZ}, \mathrm{YZ}$, and $\mathrm{DZ}$ conceived the idea and wrote the manuscript together. All authors listed have made a substantial, direct and intellectual contribution to the work, and approved it for publication.

\section{FUNDING}

This study was supported by the National Natural Science Foundation of China (31900765), Shanghai Sailing Program (19YF1441900), and China Postdoctoral Science Foundation (2018M640405). 
Grodin, E. N., Sussman, L., Sundby, K., Brennan, G. M., Diazgranados, N., Heilig, M., et al. (2018). Neural correlates of compulsive alcohol seeking in heavy drinkers. Biol. Psychiatry Cogn. Neurosci. Neuroimaging 3, 1022-1031. doi: 10.1016/j.bpsc.2018.06.009

Hanlon, C. A., Dowdle, L. T., Austelle, C. W., DeVries, W., Mithoefer, O., Badran, B. W., et al. (2015). What goes up, can come down: novel brain stimulation paradigms may attenuate craving and craving-related neural circuitry in substance dependent individuals. Brain Res. 1628, 199-209. doi: 10.1016/j.brainres.2015.02.053

Hanlon, C. A., Dowdle, L. T., Correia, B., Mithoefer, O., Kearney-Ramos, T., Lench, D., et al. (2017). Left frontal pole theta burst stimulation decreases orbitofrontal and insula activity in cocaine users and alcohol users. Drug Alcohol Depend. 178, 310-317. doi: 10.1016/j.drugalcdep.2017.03.039

Hanlon, C. A., Dowdle, L. T., Gibson, N. B., Li, X., Hamilton, S., Canterberry, M., et al. (2018). Cortical substrates of cue-reactivity in multiple substance dependent populations: transdiagnostic relevance of the medial prefrontal cortex. Transl. Psychiatry 8:186. doi: 10.1038/s41398-018-0220-9

Hanlon, C. A., Dowdle, L. T., Moss, H., Canterberry, M., and George, M. S. (2016). Mobilization of medial and lateral frontal-striatal circuits in cocaine users and controls: an interleaved tms/bold functional connectivity study. Neuropsychopharmacology 41, 3032-3041. doi: 10.1038/npp.2016.114

Hart, G., Leung, B. K., and Balleine, B. W. (2014). Dorsal and ventral streams: the distinct role of striatal subregions in the acquisition and performance of goal-directed actions. Neurobiol. Learn. Mem. 108, 104-118. doi: 10.1016/j.nlm.2013.11.003

Hu, Y., Salmeron, B. J., Gu, H., Stein, E. A., and Yang, Y. (2015). Impaired functional connectivity within and between frontostriatal circuits and its association with compulsive drug use and trait impulsivity in cocaine addiction. JAMA Psychiatry 72, 584-592. doi: 10.1001/jamapsychiatry.2015.1

Hu, Y., Salmeron, B. J., Krasnova, I. N., Gu, H., Lu, H., Bonci, A., et al. (2019). Compulsive drug use is associated with imbalance of orbitofrontal-and prelimbic-striatal circuits in punishment-resistant individuals. Proc. Natl. Acad Sci. U.S.A. 116, 9066-9071. doi: 10.1073/pnas.1819978116

Hyman, S. E., Malenka, R. C., and Nestler, E. J. (2006). Neural mechanisms of addiction: the role of reward-related learning and memory. Annu. Rev. Neurosci. 29, 565-598. doi: 10.1146/annurev.neuro.29.051605.113009

Kohno, M., Morales, A. M., Ghahremani, D. G., Hellemann, G., and London, E. D. (2014). Risky decision making, prefrontal cortex, and mesocorticolimbic functional connectivity in methamphetamine dependence. JAMA Psychiatry 71, 812-820. doi: 10.1001/jamapsychiatry.2014.399

Koob, G. F., and Le Moal, M. (1997). Drug abuse: hedonic homeostatic dysregulation. Science 278, 52-58. doi: 10.1126/science.278. 5335.52

Koob, G. F., and Volkow, N. D. (2016). Neurobiology of addiction: a neurocircuitry analysis. Lancet Psychiatry 3, 760-773. doi: 10.1016/s2215-0366(16) 00104-8

Liu, Q., Shen, Y., Cao, X., Li, Y., Chen, Y., Yang, W., et al. (2017). Either at left or right, both high and low frequency rtms of dorsolateral prefrontal cortex decreases cue induced craving for methamphetamine. Am. J. Addict. 26, 776-779. doi: 10.1111/ajad.12638

Liu, T., Li, Y., Shen, Y., Liu, X., and Yuan, T. F. (2019). Gender does not matter: add-on repetitive transcranial magnetic stimulation treatment for female methamphetamine dependents. Prog. Neuropsychopharmacol. Biol. Psychiatry 92, 70-75. doi: 10.1016/j.pnpbp.2018.12.018

Maier, S. U., Makwana, A. B., and Hare, T. A. (2015). Acute stress impairs self-control in goal-directed choice by altering multiple functional connections within the brain's decision circuits. Neuron 87, 621-631. doi: 10.1016/j.neuron.2015.07.005

McEwen, B. S., and Morrison, J. H. (2013). The brain on stress: vulnerability and plasticity of the prefrontal cortex over the life course. Neuron 79, 16-29. doi: 10.1016/j.neuron.2013.06.028

McFarland, K., and Kalivas, P. W. (2001). The circuitry mediating cocaineinduced reinstatement of drug-seeking behavior. J. Neurosci. 21, 8655-8663. doi: 10.1523/jneurosci.21-21-08655.2001

Shen, Y., Cao, X., Shan, C., Dai, W., and Yuan, T. F. (2017). Heroin addiction impairs human cortical plasticity. Biol. Psychiatry 81, e49-e50. doi: 10.1016/j.biopsych.2016.06.013

Shippenberg, T. S., and Koob, G. F. (2002). "Recent advances in animal models of drug addiction and Alcoholism," in Neuropsychopharmacology: The Fifth Generation of Progress, Vol.13, eds K. L., Davis, D. Charney, J. T. Coyle, and C. Nemeroff (Philadelphia, PA: Lippincott Williams and Wilkins), 81-97.

Strosche, A., Zhang, X., Kirsch, M., Hermann, D., Ende, G., Kiefer, F., et al. (2020). Investigation of brain functional connectivity to assess cognitive control over cue-processing in alcohol use disorder. Addict. Biol. doi: 10.1111/adb.12863. [Epub ahead of print].

The United Nations Office on Drugs and Crime. World Drug Report 2019 (2019). Available online at: https://wdr.unodc.org/wdr2019/prelaunch/ WDR19_Booklet_4_STIMULANTS.pdf (accessed April 04, 2020).

Volkow, N. D., and Fowler, J. S. (2000). Addiction, a disease of compulsion and drive: involvement of the orbitofrontal cortex. Cereb. Cortex 10, 318-325. doi: $10.1093 /$ cercor/10.3.318

Volkow, N. D., Koob, G. F., and McLellan, A. T. (2016). Neurobiologic advances from the brain disease model of addiction. N. Engl. J. Med. 374, 363-371. doi: 10.1056/NEJMra1511480

Wang, Y., Zhu, J., Li, Q., Li, W., Wu, N., Zheng, Y., et al. (2013). Altered fronto-striatal and fronto-cerebellar circuits in heroindependent individuals: a resting-state fmri study. PLOS ONE 8:e58098. doi: 10.1371/journal.pone.0058098

Zhao, D., Li, Y., Liu, T., Voon, V., and Yuan, T. F. (2020). Twice-daily theta burst stimulation of the dorsolateral prefrontal cortex reduces methamphetamine craving: a pilot study. Front. Neurosci. 14:208. doi: 10.3389/fnins.2020.00208

Conflict of Interest: The authors declare that the research was conducted in the absence of any commercial or financial relationships that could be construed as a potential conflict of interest.

Copyright (c) 2020 Zhang, Zheng, Zhang and Zhao. This is an open-access article distributed under the terms of the Creative Commons Attribution License (CC BY). The use, distribution or reproduction in other forums is permitted, provided the original author(s) and the copyright owner(s) are credited and that the original publication in this journal is cited, in accordance with accepted academic practice. No use, distribution or reproduction is permitted which does not comply with these terms. 